\title{
The abundances of nitrogen and oxygen in damped Lyman $\alpha$ systems
}

\author{
M. Pettini ${ }^{1}$, S. L. Ellison ${ }^{2}$, J. Bergeron ${ }^{3,4}$, and P. Petitjean ${ }^{4,5}$ \\ ${ }^{1}$ Institute of Astronomy, Madingley Road, Cambridge, CB3 OHA, UK \\ 2 European Southern Observatory, Casilla 19001, Santiago 19, Chile \\ ${ }^{3}$ European Southern Observatory, Karl-Schwarzschild-Strasse 2, Garching bei München, 85748, Germany \\ ${ }^{4}$ Institut d'Astrophysique de Paris, 98bis boulevard d'Arago, 75014 Paris, France \\ 5 LERMA, Observatoire de Paris, 61 avenue de l'Observatoire, 75014 Paris, France
}

Received 22 February 2002 / Accepted 28 May 2002

\begin{abstract}
We take a fresh look at the abundance of nitrogen in damped Lyman $\alpha$ systems (DLAs) with oxygen abundances between $\sim 1 / 10$ and $\sim 1 / 100$ of solar. This is a metallicity regime poorly sampled in the local universe and where QSO absorbers may hold clues to both the nucleosynthetic origin of $\mathrm{N}$ and the chemical evolution of high redshift galaxies. We combine new VLT UVES observations with others from the literature to form a sample of 10 DLAs in which the abundances of $\mathrm{N}$ and of one of the two $\alpha$-capture elements $\mathrm{O}$ or $\mathrm{S}$ have been measured. The sample consists exclusively of high resolution, high signal-to-noise ratio data obtained with 8-10 m class telescopes. We confirm earlier reports that the (N/O) ratio exhibits a larger range of values than other ratios of heavy elements in DLAs; however, all 10 DLAs fall in the region of the $(\mathrm{N} / \mathrm{O}) \mathrm{vs}$. $(\mathrm{O} / \mathrm{H})$ plot delimited by the primary and secondary levels of nitrogen production. Our results provide empirical evidence in support of the proposal that intermediate mass stars $\left(4 \lesssim M / M_{\odot} \lesssim 7\right)$ are the main source of primary nitrogen, so that its release into the interstellar medium lags behind that of oxygen, which is produced by Type II supernovae. A high proportion (40\%) of the DLAs in our sample have apparently not yet attained the full primary level of $\mathrm{N}$ enrichment; this finding may be an indication that the metallicity regime we are studying preferentially picks out galaxies which have only recently condensed out of the intergalactic medium and begun forming stars. Alternatively, the delay in the release of $\mathrm{N}$ following an episode of star formation may increase with decreasing metallicity, if stars of progressively lower masses than $4 M_{\odot}$ can synthesize $\mathrm{N}$ in their hotter interiors. In this general picture, the uniform value of (N/O) measured locally in metal-poor star-forming galaxies implies that they are not young, and is consistent with the presence of older stellar populations revealed by imaging studies with the Hubble Space Telescope.
\end{abstract}

Key words. cosmology: observations - galaxies: abundances - galaxies: ISM - quasars: absorption lines

\section{Introduction}

The nucleosynthetic origin of nitrogen continues to be a subject of considerable interest and discussion. There is general agreement that the main pathway is a six step process in the $\mathrm{CN}$ branch of the $\mathrm{CNO}$ cycle which takes place in the stellar $\mathrm{H}$ burning layer, with the net result that ${ }^{14} \mathrm{~N}$ is synthesised from ${ }^{12} \mathrm{C}$ and ${ }^{16} \mathrm{O}$. The continuing debate, however, centres on which range of stellar masses is responsible for the bulk of the nitrogen production. A comprehensive reappraisal of the problem was presented by Henry et al. (2000) who compiled an extensive set of abundance measurements and computed chemical evolution models using published yields. Briefly, nitrogen has both a primary and a secondary component, depending on whether the seed carbon and oxygen are those manufactured by the star during helium burning, or were already present when the star first condensed out of the interstellar medium (ISM).

Send offprint requests to: M. Pettini,

e-mail: pettini@ast.cam.ac.uk

\subsection{Nitrogen and oxygen in $\mathrm{H} I /$ regions}

Observational evidence for this dual nature of nitrogen is provided mainly from measurements of the $\mathrm{N}$ and $\mathrm{O}$ abundances in H II regions. (For simplicity in this paper we use parentheses to indicate logarithmic ratios of number densities; adopting the recent reappraisal of solar photospheric abundances by Holweger 2001, we have $(\mathrm{N} / \mathrm{H})_{\odot}=-4.07 ;(\mathrm{O} / \mathrm{H})_{\odot}=-3.26$; and $(\mathrm{N} / \mathrm{O})_{\odot}=-0.81$.) In $\mathrm{H}$ II regions of nearby galaxies, $(\mathrm{N} / \mathrm{O})$ exhibits a strong dependence on $(\mathrm{O} / \mathrm{H})$ when the latter is greater than $\sim 2 / 5$ solar; this is generally interpreted as the regime where secondary $\mathrm{N}$ becomes dominant ${ }^{1}$. At low metallicities on the other hand, when $(\mathrm{O} / \mathrm{H}) \lesssim-4.0$ (that is, $\lesssim 1 / 5$ solar), $\mathrm{N}$ is mostly primary and tracks $\mathrm{O}$; this results in a plateau at $(\mathrm{N} / \mathrm{O}) \simeq-1.5$.

\footnotetext{
${ }^{1}$ As an aside, Henry et al. (2000) pointed out that the rise in (N/O) with $(\mathrm{O} / \mathrm{H})$ is steeper than would be normally expected for a purely secondary element and proposed that the effect is augmented by a decreasing O yield with increasing metallicity (Maeder 1992).
} 
Table 1. Damped Ly $\alpha$ systems observed.

\begin{tabular}{lcccccccc}
\hline \hline QSO & $\begin{array}{c}V \\
(\mathrm{mag})\end{array}$ & $z_{\mathrm{em}}$ & $z_{\text {abs }}$ & $\begin{array}{c}N(\mathrm{H} \mathrm{I})^{a} \\
\left(\mathrm{~cm}^{-2}\right)\end{array}$ & $\begin{array}{c}\text { Exp. Time } \\
(\mathrm{s})\end{array}$ & $\begin{array}{c}\text { Resolution } \\
\left(\mathrm{km} \mathrm{s}^{-1}\right)\end{array}$ & $\begin{array}{c}\text { Wavelength Range } \\
(\AA)\end{array}$ & $S / N^{b}$ \\
\hline Q1409+095 & 18.6 & 2.856 & 2.45620 & $3.5 \times 10^{20}$ & 16200 & 7 & $3793-4989,6726-10255^{c}$ & $15-25$ \\
Q1444+014 & 18.5 & 2.206 & 2.08681 & $1.6 \times 10^{20}$ & 18000 & 7 & $3295-6650^{d}$ & $10-20$ \\
Q2206-199 & 17.33 & 2.559 & 2.07623 & $2.7 \times 10^{20}$ & 24300 & 7 & $3295-10255^{d}$ & $20-25$ \\
\hline
\end{tabular}

${ }^{a}$ Determined by profile fitting to the damping wings of the Lyman $\alpha$ absorption line (see Sect. 2 and Fig. 1). Typical error in $N(\mathrm{H} \mathrm{I})$ is $\pm 10 \%$.

${ }^{b}$ The signal-to-noise ratio varies along each spectrum. These values (per pixel) refer to the QSO continuum in the regions of the N I $\lambda \lambda 1200$ and $\mathrm{O} I$ I $\lambda 1302.2$ lines.

${ }^{c}$ With a wavelength gap between 8520 and $8667 \AA$.

${ }^{d}$ With some wavelength gaps.

The principal sources of primary $\mathrm{N}$ are thought to be intermediate mass stars $\left(4 \lesssim M / M_{\odot} \lesssim 7\right.$ ) during the asymptotic giant branch (AGB) phase. Henry et al. (2000) showed that, integrating along the IMF the $\mathrm{N}$ yields from intermediate mass stars by van den Hoek \& Groenewegen (1997) and the O yields from massive stars by Maeder (1992) at metallicity $Z=1 / 20 Z_{\odot}$, one obtains $(\mathrm{N} / \mathrm{O})=-1.41$ in good agreement with the observed plateau.

A corollary of the hypothesis that intermediate mass stars are the main producers of primary nitrogen is that its release in the ISM should lag behind that of $\mathrm{O}$, since the latter is widely believed to be produced by massive stars which explode as Type II supernovae (SN) soon after an episode of star formation. Henry et al. (2000) calculated this time delay to be approximately $250 \mathrm{Myr}$; at low metallicities the $(\mathrm{N} / \mathrm{O})$ ratio could then perhaps be used as a clock with which to measure the past rate of star formation, as proposed by Edmunds \& Pagel (1978). Specifically, in metal-poor galaxies which have only recently experienced a burst of star formation one may expect to find values of $(\mathrm{N} / \mathrm{O})$ below the primary plateau at $(\mathrm{N} / \mathrm{O}) \simeq-1.5$.

This scenario has been discussed at length over the last few years with claims of both conflicting and supporting evidence from the data. Izotov \& Thuan (1999) and Izotov et al. (2001) were struck by the constant values of all the element ratios, including $(\mathrm{N} / \mathrm{O})$, which they measured in blue compact dwarf $(\mathrm{BCD})$ galaxies with $(\mathrm{O} / \mathrm{H}) \lesssim-4.4$ (corresponding to an oxygen abundance less than $\sim 1 / 15$ of solar). This finding led them to the radical proposal that (a) these are galaxies undergoing their first burst of star formation, and (b) all the elements they observed have a primary origin in massive stars, so that they are released at the same time as $\mathrm{O}$, thereby disposing altogether of the notion of a time delay. On the other hand, a variety of studies using high resolution Hubble Space Telescope (HST) images have shown that many of these BCDs host old stellar populations, with ages greater than $1 \mathrm{Gyr}$ (e.g. SchulteLadbeck et al. 2001 and references therein; Crone et al. 2002). Generally, these low mass galaxies tend to have low rates of star formation (even though they are observed during a bursting phase). If the lag between $\mathrm{O}$ and $\mathrm{N}$ production is only $250 \mathrm{Myr}$, as proposed by Henry et al. (2000), it is perhaps not surprising that most of them lie near the primary value of the $(\mathrm{N} / \mathrm{O})$ ratio expected from intermediate mass stars (Pilyugin 1999).

In contrast, a recent survey by Contini et al. (2002) found that UV-selected galaxies at intermediate redshifts $(0<z<0.4)$ exhibit a wide range of $(\mathrm{N} / \mathrm{O})$ values at a given $(\mathrm{O} / \mathrm{H})$, in many cases well below the primary level. While Contini et al. interpret their results as evidence in favour of a delayed production of primary $\mathrm{N}$, one may well wonder at the high fraction of galaxies apparently caught within $250 \mathrm{Myr}$ since the last major episode of oxygen enrichment. Possibly the UV-continuum selection technique strongly favours galaxies with high rates of star formation in the recent past.

\subsection{Nitrogen and oxygen in QSO absorption line systems}

As pointed out by Pettini et al. (1995), clues to the nucleosynthetic origin of nitrogen can also be provided by QSO absorption line systems, particularly the high column density $\left(N(\mathrm{H} \mathrm{I}) \geq 2 \times 10^{20} \mathrm{~cm}^{-2}\right.$ ) damped Lyman $\alpha$ absorbers (DLAs). These are thought to represent an early stage in the evolution of galaxies, when most of their baryonic mass was in the interstellar medium. Apart from the obvious interest in measuring element abundances in the distant past, when galaxies were young, one of the advantages of DLAs is that they are generally of low metallicity, approximately between $1 / 10$ and $1 / 100$ solar (Pettini et al. 1999; Prochaska \& Wolfe 2002). Thus, they probe a regime where local H II region abundance measurements are sparse or non-existent and where the effect of a delayed production of primary nitrogen should be most pronounced.

There are practical difficulties, however, in measuring (N/O) in DLAs. The resonance lines most easily accessible, the N I triplet $\lambda \lambda 1199.5,1200.2,1200.7$ and O I $\lambda 1302.2$, have very different optical depths. While the latter is normally saturated in DLAs, the former is weak and can be difficult to detect, particularly as it falls within the Lyman $\alpha$ forest. Pettini et al. (1995), and subsequently Lu et al. (1998) and Centurión et al. (1998), attempted to circumvent the first problem by using the abundances of other $\alpha$-capture elements, such as $\mathrm{S}$ and $\mathrm{Si}$, as proxies for $(\mathrm{O} / \mathrm{H})$. These studies found that the $(\mathrm{N} / \alpha)$ ratio in DLAs spans a wide range of values, broadly within the range bracketed by the predictions for primary and secondary production of nitrogen. However, these results have been criticized by Izotov \& Thuan (1999) and more recently Izotov et al. (2001). Unlike O and N, which are mostly neutral in H I gas, S and $\mathrm{Si}$ are singly ionised. Since in principle S II and Si II can also occur in H II regions, Izotov and collaborators have proposed that unaccounted ionisation corrections, rather than real 
abundance variations, are the cause of the observed scatter of the $(\mathrm{N} / \alpha)$ ratios in DLAs. Their model, however, makes the extreme assumption that essentially all the metals are located in (presumably self-enriched) H II regions, and that the metallicity of the neutral phase is negligible.

\subsection{Motivations for the present study}

In an attempt to clarify this confusing state of affairs, we have begun a new programme of observations with the Ultraviolet-Visual Echelle Spectrograph (UVES) on the VLT (Kueyen) telescope (Dekker et al. 2000). In order to minimize the practical difficulties outlined above, we targeted DLAs which (a) have relatively low values of hydrogen column density $\left(N(\mathrm{H} \mathrm{I}) \lessgtr 4 \times 10^{20} \mathrm{~cm}^{-2}\right.$ ), thus increasing the probability that the O I $\lambda 1302.2$ line may not be strongly saturated, and (b) are at relatively low redshifts $\left(z_{\mathrm{abs}} \lesssim 2.7\right)$, where the Lyman $\alpha$ forest begins to thin out. Despite this observing strategy, which capitalises on the superior performance of UVES at ultraviolet wavelengths (D'Odorico et al. 2000), our endeavours have been only partially successful, as we shall see. In addition, we bring together a number of relevant abundance measurements which have been published since the surveys by Lu et al. (1998) and Centurión et al. (1998) for a comprehensive reappraisal of the abundances of oxygen and nitrogen in DLAs.

\section{Observations and data reduction}

Details of the DLAs observed are collected in Table 1. The data were secured over the three nights of 28-30 May 2000. By using different cross-disperser gratings and dichroic filters we recorded the spectra of three QSOs over the wavelength ranges listed in the penultimate column of Table 1 . With a 1 arcsec wide entrance slit and $2 \times 2$ binning on the CCDs, the resulting spectral resolution was between 6.7 and $7.1 \mathrm{~km} \mathrm{~s}^{-1}$ FWHM, measured from the widths of emission lines of the ThAr hollow-cathode lamp used for wavelength calibration. The observations consisted of a number of one hour long exposures; the QSOs were moved along the slit between exposures so as to use different portions of the detectors to record the spectrum of each object. The slit was aligned at the parallactic angle throughout the observations.

The two-dimensional images were processed with the UVES standard pipeline software; see for example Ellison et al. (2001) for a description of the main steps. The individual spectra extracted for each QSO were mapped onto a common, linear, vacuum heliocentric, wavelength scale; the interval between successive wavelength bins was set to the average value of the original CCD pixels, typically $\delta \lambda=0.03 \AA$ and $0.04 \AA$ in the blue and red wavelength regions respectively. The individual extractions were then co-added, with weights proportional to the $S / N$ ratios, to produce final blue and red spectra for each QSO. With several independent exposures at our disposal, we were able to exclude from the sum wavelength bins which deviated from the mean by several standard deviations, because of residuals from the subtraction of cosmic-ray events, strong sky emission lines, and other invalid pixels.
Figure 1 shows portions of the final spectra in the regions near the damped Lyman $\alpha$ lines at $z_{\mathrm{abs}}=2.45620$ in $\mathrm{Q} 1409+095, z_{\mathrm{abs}}=2.08681$ in Q1444+014, and $z_{\mathrm{abs}}=2.07623$ in Q2206-199, after division by the underlying QSO continuum. Superposed on the data are theoretical damped absorption profiles corresponding to the values of $N(\mathrm{H} \mathrm{I})$ listed in Table 1; the typical error in the determination of $N(\mathrm{H} \mathrm{I})$ is about $10 \%$. Our observations revealed the existence of a second high column density absorption system in Q1409+095, at $z_{\text {abs }}=2.66820$. For completeness, we have included it in Fig. 1; the profile fit shown is for $N(\mathrm{H} \mathrm{I})=5 \times 10^{19} \mathrm{~cm}^{-2}$.

\section{Metal absorption lines in the four DLAs}

\subsection{Rationale}

As is the case with modern echelle spectra, our observations of the three QSOs in Table 1 provide a wealth of information on several absorption systems, as well as on the Lyman $\alpha$ forest. In the present study we are primarily interested in the abundances of nitrogen and oxygen, detected respectively via the N I $\lambda \lambda 1199.5,1200.2,1200.7$ triplet and the O I $\lambda 1302.2$ line. Also of relevance to our analysis are the abundances of silicon (measured via Si II 21304.4 ) and iron (several absorption lines of Fe II throughout the spectral regions covered). Silicon is an $\alpha$-capture element which can provide an additional check on the oxygen abundance in cases where the O I $\lambda 1302$ line is saturated and the corresponding value of $N(\mathrm{O} \mathrm{I})$ is uncertain. Sulphur would also be useful in this context, but unfortunately the relevant absorption lines are either blended or undetected in most of the cases considered here. Iron is of interest because it is thought to be produced primarily by Type Ia supernovae; like nitrogen, its release into the interstellar medium should be delayed relative to that of oxygen. The four elements in question, $\mathrm{N}, \mathrm{O}, \mathrm{Si}$, and $\mathrm{Fe}$, respond differently to depletion onto dust grains. In the local ISM, $\mathrm{N}$ and $\mathrm{O}$ are normally present in the gas phase with near-solar abundances; $\mathrm{Si}$ and $\mathrm{Fe}$, on the other hand, are susceptible to dust depletion, the latter normally more than the former (e.g. Savage \& Sembach 1996). Thus, by considering all four elements together, it may be possible to decouple nucleosynthesis and depletion effects.

\subsection{Profile fitting and abundance determinations}

Normalised profiles of the absorption lines mentioned above were fitted with Voigt profiles using the VPFIT package ${ }^{2}$. VPFIT deconvolves the composite absorption profiles into the minimum number of discrete components and returns for each the most likely values of redshift $z$, Doppler width $b$ $\left(\mathrm{km} \mathrm{s}^{-1}\right)$, and column density $N\left(\mathrm{~cm}^{-2}\right)$ by minimizing the difference between observed and computed profiles. The profile decomposition takes into account the instrumental point spread function of UVES. As we shall see shortly, the velocity structure of three out of the four DLAs studied here is very simple. In each case the same set of values of $z$ and $b$ was found to provide a satisfactory fit to the absorption

\footnotetext{
2 VPFIT is available at http://www. ast.cam.ac.uk/ rfc/ vpfit.html
} 

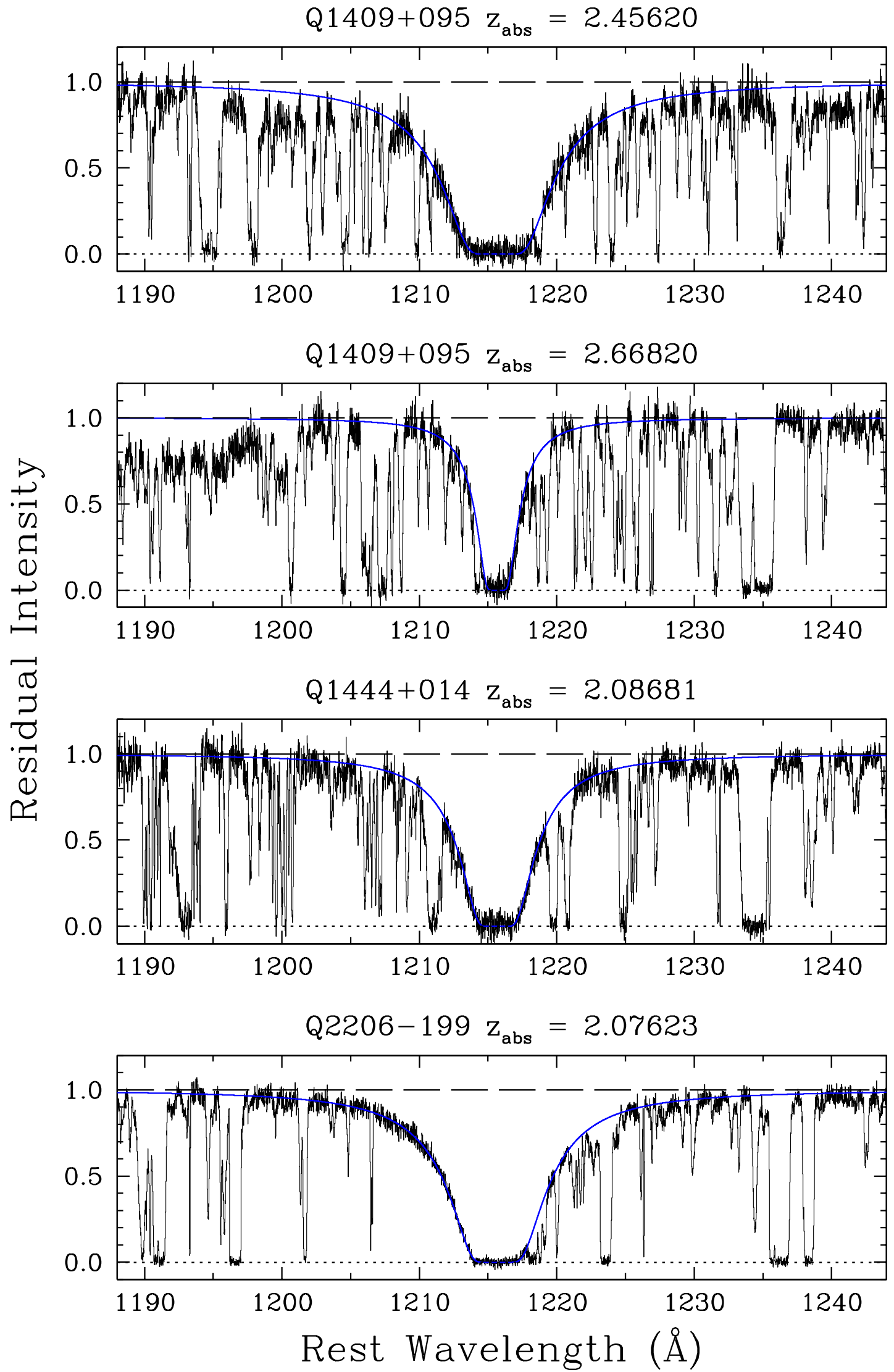

Fig. 1. Normalised UVES spectra of the three QSOs observed showing the regions of the four damped Lyman $\alpha$ lines. Superposed on the data are theoretical absorption profiles for Lyman $\alpha$ lines with the neutral hydrogen column densities $N(\mathrm{H} \mathrm{I})$ listed in Table 1. 
Table 2. Component structure of the metal absorption lines.

\begin{tabular}{lccccccc}
\hline \hline QSO & $\begin{array}{c}\text { Component } \\
\text { Number }\end{array}$ & $z_{\text {abs }}$ & $\begin{array}{c}b \\
\left(\mathrm{~km} \mathrm{~s}^{-1}\right)\end{array}$ & $\begin{array}{c}\log N(\mathrm{O} \mathrm{I}) \\
\left(\mathrm{cm}^{-2}\right)\end{array}$ & $\begin{array}{c}\log N(\mathrm{Si} \text { II }) \\
\left(\mathrm{cm}^{-2}\right)\end{array}$ & $\begin{array}{c}\log N(\mathrm{Fe} \mathrm{II}) \\
\left(\mathrm{cm}^{-2}\right)\end{array}$ & $\begin{array}{c}\log N(\mathrm{~N} \mathrm{I}) \\
\left(\mathrm{cm}^{-2}\right)\end{array}$ \\
\hline Q1409+095 & 1 & 2.45597 & 12.9 & $14.76 \pm 0.1$ & $13.82 \pm 0.03$ & $13.46 \pm 0.03$ & $\leq 12.80$ \\
& 2 & 2.45630 & 5.8 & $14.27 \pm 0.03$ & $13.08 \pm 0.03$ & $12.81 \pm 0.03$ & $\leq 12.50$ \\
& 3 & 2.45648 & 7.3 & $14.82 \pm 0.1$ & $13.63 \pm 0.03$ & $13.29 \pm 0.03$ & $\leq 12.77$ \\
Q1409+095 & 1 & 2.66820 & 7.5 & $15.27 \pm 0.1$ & $14.02 \pm 0.03$ & $13.85 \pm 0.03$ & $\leq 13.45$ \\
& & & & & & & \\
Q2206-199 & 1 & 2.07623 & 6.1 & $15.24 \pm 0.09$ & $13.65 \pm 0.02$ & $13.37 \pm 0.02$ & $\leq 12.88$ \\
\hline
\end{tabular}

lines of O I, Si II, and Fe II. Oscillator strengths and rest wavelengths were adopted from the up to date compilation of atomic parameters maintained by J. X. Prochaska at http://kingpin.ucsd.edu/ hiresdla/atomic.dat (see also Prochaska et al. 2001). The solutions returned by VPFIT are collected in Table 2.

The error estimates $\sigma(\log N)$ returned by VPFIT were typically $<0.05$ dex for the optically thin Si II and Fe II lines and $<0.1$ dex for the saturated O I lines. To test whether these errors are realistic we performed Monte Carlo type simulations similar to those described by Bowen et al. (1995) and Bowen et al. (2002). Briefly, for each DLA we used as the starting point the theoretical spectrum produced by VPFIT with the best fit parameters given in Table 2. From this template we generated 300 spectra with the same $S / N$ as the original data; these were then refitted to deduce values of $b$ and $N$. From Gaussian fits to the distributions of 300 values of $b$ and $N$ we determined values of $\sigma(b)$ and $\sigma(\log N)$. The former are typically $\pm 0.2 \mathrm{~km} \mathrm{~s}^{-1}$; the latter, which are comparable to the values returned by VPFIT, are listed in Table 2. Despite the good agreement between the two methods, it must be remembered that these errors reflect primarily the $S / N$ of the data and do not include the possibility that the velocity dispersion parameter $b$ may be different between the different ions considered. The consequences of letting $b$ vary from ion to ion are discussed below for each DLA individually.

The species observed are the major ionisation stages of their respective elements in $\mathrm{H}$ I regions. Thus, to obtain their abundances relative to hydrogen we simply divide the column densities returned by VPFIT by $N(\mathrm{H} \mathrm{I}$ ) (after adding together the values for individual velocity components within the same DLA, when more than one component is present). Finally these values are referred to the solar abundance scale (Holweger 2001). The results are collected in Table 3 , where the solar reference values are listed in the footnotes.

This procedure would overestimate the abundances of $\mathrm{Si}$ and $\mathrm{Fe}$ if some of the Si II and Fe II absorption we detect were due to ionised gas associated with the neutral DLA. Conversely, the abundances of $\mathrm{O}$ and $\mathrm{N}$ would be underestimated if these elements were partly ionised in the H I region. In the cases under consideration, however, there is little evidence that these reservations are justified. First, all four species exhibit very similar velocity structure; we see no indication of components present in the first ions and missing in the neutrals, or vice versa. Second, the recent reappraisal of this problem by
Vladilo et al. (2001) shows that at the values of $N(\mathrm{H} \mathrm{I})$ of the DLAs studied here, the ionisation corrections to the abundances determined as above are smaller than the typical error of \pm 0.15 dex of our abundance estimates. We now briefly comment on each DLA in turn.

\section{3. $T$ The $z_{a b s}=2.45620 D L A$ in $Q 1409+095$}

The Si II $\lambda 1304.4$ and Fe II $\lambda 2344.2$ lines in this DLA are unsaturated and are well reproduced with three absorption components spanning a velocity range $\Delta v=44 \mathrm{~km} \mathrm{~s}^{-1}$ (see Fig. 2 and Table 2). This is a very metal-poor system. Adding together the column densities for the three components we deduce that the abundance of $\mathrm{Si}$ is $1 / 100$ of solar, and that of Fe only little more than $1 / 200$ of solar (see Table 3 ).

Even at these low abundances, and with a hydrogen column density of $3.5 \times 10^{20} \mathrm{~cm}^{-2}$ (near the lower limit of $2 \times 10^{20} \mathrm{~cm}^{-2}$ commonly adopted as the definition of a DLA), the O I $\lambda 1302.2$ line is saturated (see Fig. 2). If we adopt the same set of absorption parameters as for Si II and Fe II, we deduce an oxygen abundance of $1 / 135$ of solar, in reasonable agreement with that of Si. The good model fit to O I $\lambda 1302.2$ makes it unlikely that we have overestimated $N(\mathrm{O} \mathrm{I})$ (the line would be broader than observed). The oxygen abundance could be higher if our model underestimates the saturation of the O I $\lambda 1302.2$ line; this possibility, however, does not alter the interpretation of our results below because it would tend to emphasize the "anomalously" low (N/O) ratio (see Sect. 5).

The N I $\lambda 1200$ triplet at $z_{\mathrm{abs}}=2.45620$ falls within the Lyman $\alpha$ forest of this $z_{\mathrm{em}}=2.856$ QSO. Figure 2 shows the best fit to this spectral region obtained with the model parameters of the other three lines, but it is difficult to be confident that the weak absorption we see, particularly in the $\lambda 1200.2$ transition, is actually N I and not a Lyman $\alpha$ forest line. Consequently, we prefer to adopt the corresponding $N(\mathrm{~N} \mathrm{I})$ as an upper limit to the column density of $\mathrm{N} \mathrm{I}$, from which we deduce that the abundance of $\mathrm{N}$ is less than $1 / 2000$ of solar.

\section{4. $T$ The $z_{a b s}=2.66820$ sub-DLA in $Q 1409+095$}

There is a second high column density system in Q1409+095; although its $N(\mathrm{H} \mathrm{I})=5.0 \times 10^{19} \mathrm{~cm}^{-2}$ is below the limit normally adopted for DLAs (such systems are sometimes referred to as sub-DLAs), the damping wings of the Lyman $\alpha$ line are easily recognisable in the UVES spectrum (see Fig. 1) and $N(\mathrm{H} \mathrm{I})$ is well determined. 
Table 3. Ion column densities and element abundances.

\begin{tabular}{|c|c|c|c|c|c|c|c|c|c|c|}
\hline QSO & $z_{\mathrm{abs}}$ & $\begin{array}{c}\log N(\mathrm{H} \mathrm{I})^{a} \\
\left(\mathrm{~cm}^{-2}\right)\end{array}$ & $\begin{array}{c}\log N(\mathrm{O} \mathrm{I}) \\
\left(\mathrm{cm}^{-2}\right)\end{array}$ & $\begin{array}{c}\log N(\mathrm{Si} \text { II }) \\
\left(\mathrm{cm}^{-2}\right)\end{array}$ & $\begin{array}{c}\log N(\mathrm{Fe} \text { II }) \\
\left(\mathrm{cm}^{-2}\right)\end{array}$ & $\begin{array}{c}\log N(\mathrm{~N} \mathrm{I}) \\
\left(\mathrm{cm}^{-2}\right)\end{array}$ & {$[\mathrm{O} / \mathrm{H}]^{b}$} & {$[\mathrm{Si} / \mathrm{H}]^{b}$} & {$[\mathrm{Fe} / \mathrm{H}]^{b}$} & {$[\mathrm{~N} / \mathrm{H}]^{b}$} \\
\hline Q1409+095 & 2.45620 & 20.54 & $15.15 \pm 0.06$ & $14.08 \pm 0.02$ & $13.74 \pm 0.02$ & $\leq 13.19$ & -2.13 & -2.00 & -2.25 & $\leq-3.28$ \\
\hline Q1409+095 & 2.66820 & 19.70 & $15.27 \pm 0.1$ & $14.02 \pm 0.03$ & $13.85 \pm 0.03$ & $\leq 13.45$ & -1.17 & -1.22 & -1.30 & $\leq-2.18$ \\
\hline Q2206-199 & 2.07623 & 20.44 & $15.24 \pm 0.09$ & $13.65 \pm 0.02$ & $13.37 \pm 0.02$ & $\leq 12.88$ & -1.94 & -2.33 & -2.52 & $\leq-3.49$ \\
\hline
\end{tabular}

${ }^{a}$ Typical error in $\log N(\mathrm{H} \mathrm{I})$ is \pm 0.04 .

${ }^{b}$ In the usual notation: $[\mathrm{X} / \mathrm{H}]=\log (\mathrm{X} / \mathrm{H})-\log (\mathrm{X} / \mathrm{H})_{\odot}$. Solar abundances are from the recent updates by Holweger $(2001): \log (\mathrm{O} / \mathrm{H})_{\odot}=-3.264$; $\log (\mathrm{Si} / \mathrm{H})_{\odot}=-4.464 ; \log (\mathrm{Fe} / \mathrm{H})_{\odot}=-4.552 ;$ and $\log (\mathrm{N} / \mathrm{H})_{\odot}=-4.069$.
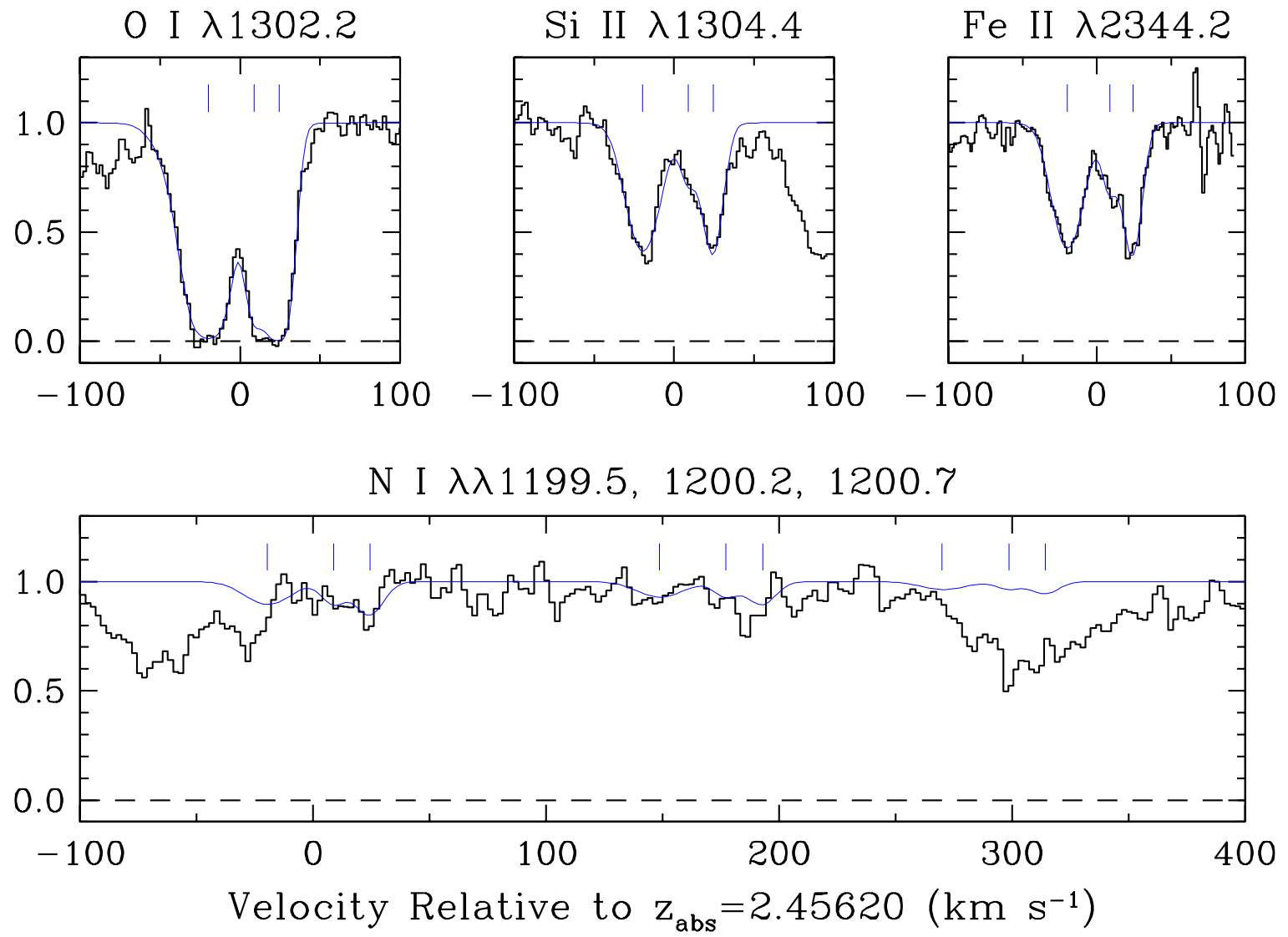

Fig. 2. Normalised absorption profiles (histograms) of selected metal lines in the $z_{\text {abs }}=2.45620$ DLA in Q1409+095. The $y$-axis is residual intensity. The thin continuous lines show theoretical profiles produced by VPFIT with the parameters listed in Table 2 . Three velocity components, indicated by vertical tick marks, contribute to the absorption in this DLA.

The O I, Si II, and Fe II absorption lines in this system are well reproduced with a single absorption component with a narrow velocity dispersion, $b=7.5 \mathrm{~km} \mathrm{~s}^{-1}$ (see Fig. 3); although there may be further substructure within this component, it is difficult to resolve it unambiguously in our data. All three lines give consistent abundances of between $1 / 15$ and $1 / 20$ of solar (see Table 3). Again, the N I triplet is blended with Lyman $\alpha$ forest absorption. While there are features which match the expected positions of two of the N I lines, we take the fit shown in Fig. 3 as indicative of the maximum amount of $\mathrm{N} \mathrm{I}$ which could be present. In this case, $\mathrm{N}$ is less abundant than $1 / 150$ of solar.

\subsection{The $Z_{a b s}=2.08681$ DLA in $Q 1444+014$}

Unlike the other DLAs in this study, this system exhibits a very complex velocity structure. The fit to the Si II $\lambda 1304.4$ line requires at least 13 components spread over the velocity 

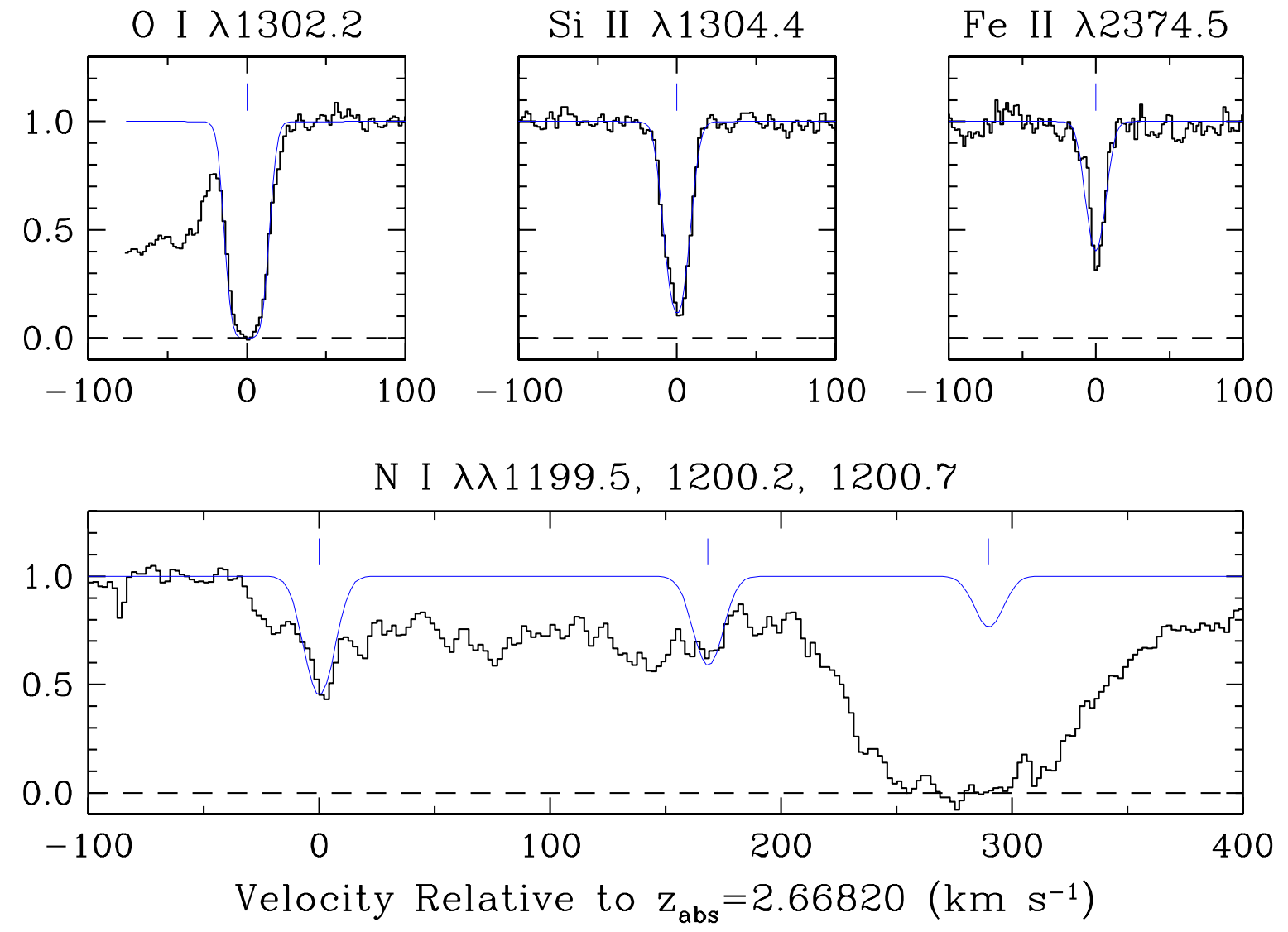

Fig. 3. Normalised absorption profiles (histograms) of selected metal lines in the $z_{\text {abs }}=2.66820$ sub-DLA in Q1409+095. The $y$-axis is residual intensity. The thin continuous lines show theoretical profiles produced by VPFIT with the parameters listed in Table 2 . The fit to the N I region (within the Lyman $\alpha$ forest) indicates the maximum amount of N I which could be present.

interval $\Delta v \simeq 350 \mathrm{~km} \mathrm{~s}^{-1}$, from -170 to $+176 \mathrm{~km} \mathrm{~s}^{-1}$ relative to $z_{\mathrm{abs}}=2.08681$ (see Fig. 4). Unfortunately, many of the components are strongly saturated in $\mathrm{O} I$; while the weaker ones are well matched by the model parameters returned by VPFIT for $\mathrm{Si}$ II, we cannot determine a reliable value of the total column density of $\mathrm{O}$ I as it is dominated by the heavily saturated absorption between $v \simeq 0$ and $-150 \mathrm{~km} \mathrm{~s}^{-1}$. In the bottom panel we show the partial fit, by the weaker components only, to the region encompassing the $\mathrm{N}$ I triplet. The combination of $13 \mathrm{ab}-$ sorption components which partially overlap in the closely separated triplet lines, together with blending with Lyman $\alpha$ forest lines, makes it impossible to estimate the abundance of N. As we are unable to determine (N/O) in this system, we do not consider it further in this paper.

\subsection{The $z_{a b s}=2.07623$ DLA in Q2206-199}

This is a well-known DLA, one of the first to be studied at echelle spectral resolution thanks to the brightness of the QSO (Pettini et al. 1990; Pettini \& Hunstead 1990). It has a remarkably simple velocity structure - the absorption apparently arises in a single component with $b \simeq 6 \mathrm{~km} \mathrm{~s}^{-1}$ (Fig. 5 and Table 2). This property makes it one of the few DLAs suitable for measuring the abundance of deuterium (Pettini \& Bowen 2001). It also exhibits a very low metallicity; O, Si and Fe are below solar by factors of 90, 210, and 330 respectively
(Table 3). For the elements in common ( $\mathrm{Si}$ and $\mathrm{Fe}$ ), our results are in good agreement (within 0.1 dex) with the Keck data analysed by Prochaska \& Wolfe (1997). As can be seen from Fig. 5, we have a tentative detection of $\mathrm{N}$ I from which we deduce an upper limit to the abundance of $\mathrm{N}$ of only $3 \times 10^{-4}$ of solar.

It is interesting that in this system we deduce $[\mathrm{O} / \mathrm{Si}]=$ $+0.4 \pm 0.1$ (Table 3 ). Possibly, this is a hint of the trend of increasing $[\mathrm{O} / \mathrm{Si}]$ with decreasing metallicity seen in Galactic halo stars with $[\mathrm{Fe} / \mathrm{H}] \lesssim-2$ (Edvardsson et al. 1993; Ryan et al. 1996; Israelian et al. 2001). Less interesting explanations are that $\mathrm{Si}$ is depleted onto dust (although a depletion by a factor of 0.4 in the log seems somewhat high, given the low metallicity of this DLA - see Pettini et al. 1997) or that we have overestimated $N(\mathrm{O}$ I) when fitting the saturated $\lambda 1302$ absorption line. The abundance of $\mathrm{S}$, an undepleted element which tracks O more closely than Si (Israelian \& Rebolo 2001; Takada-Hidai et al. 2002 - see discussion in Sect. 4) would help discriminate between these three possibilities, but unfortunately all three transitions of the S II $\lambda 1256$ multiplet in this system are blended with Lyman $\alpha$ forest lines.

\section{The DLA sample}

In the last few years there have been several new measurements of the abundance of $\mathrm{N}$, generally reported as part of abundance studies of individual DLAs. We have collected these data in 


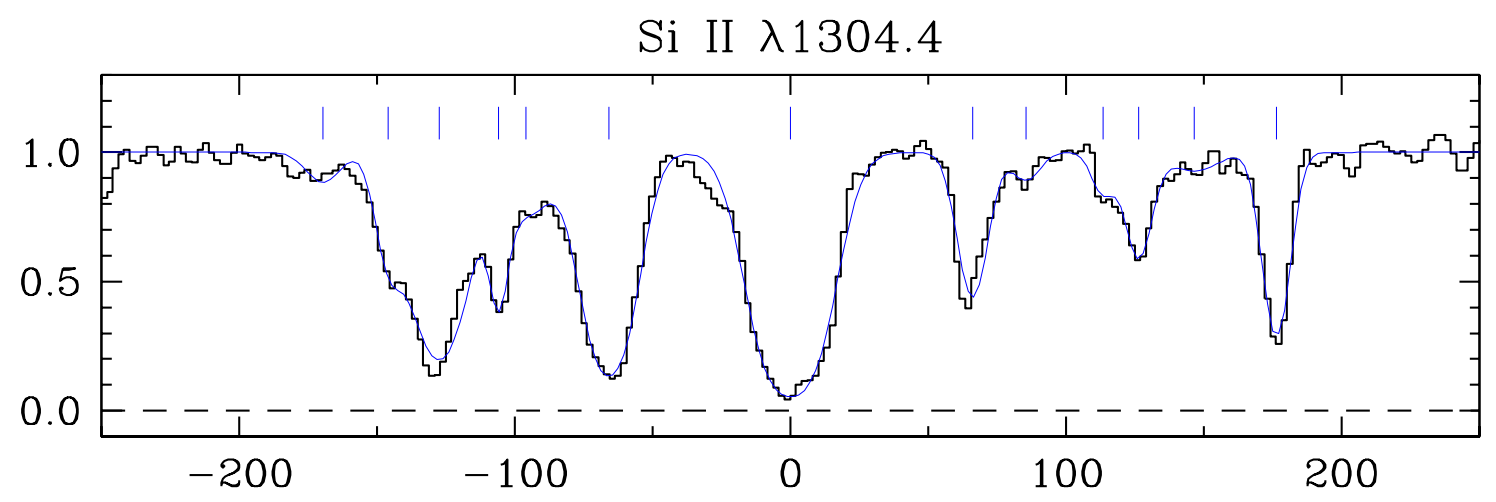

O I $\lambda 1302.2$
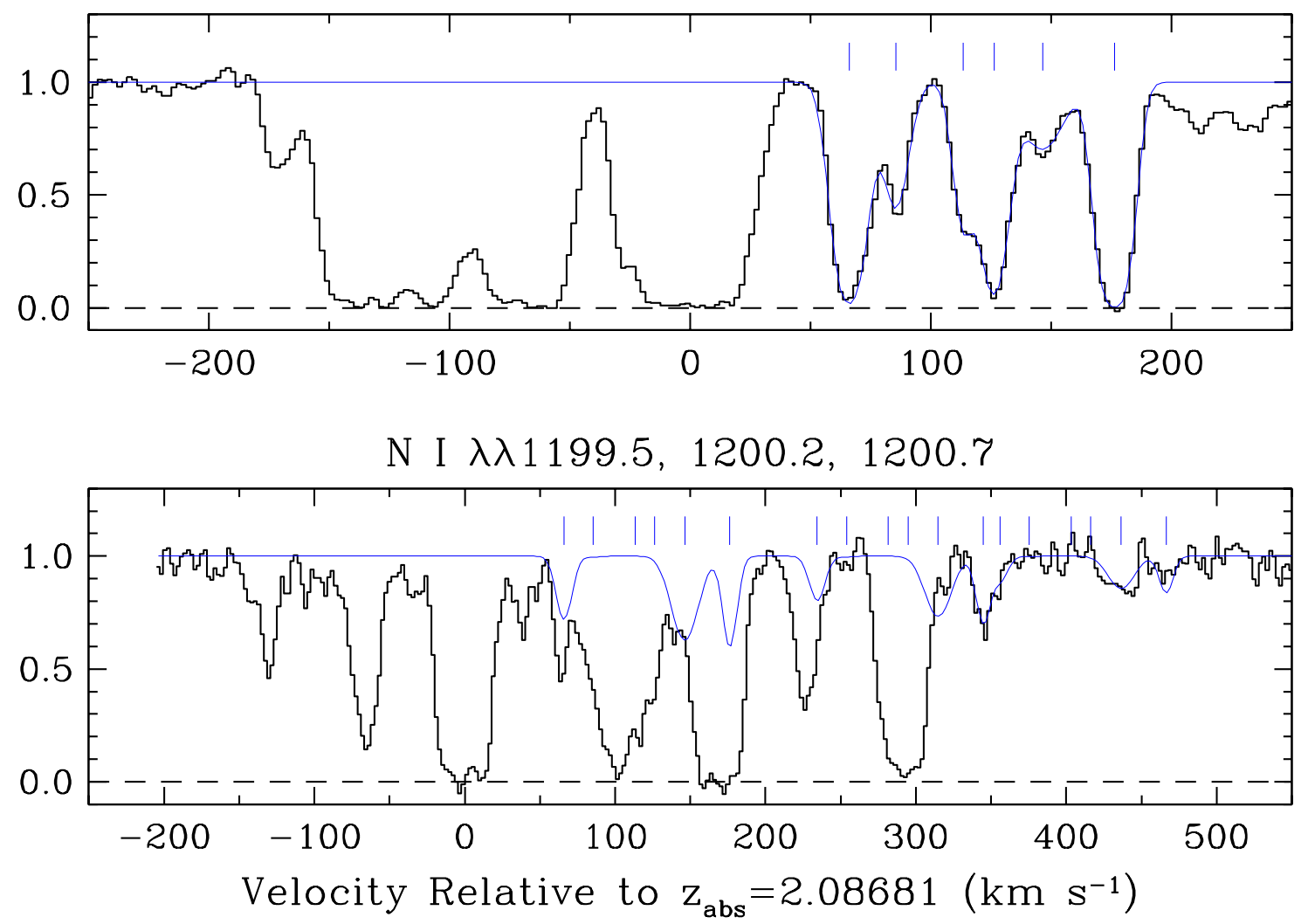

Fig. 4. Normalised absorption profiles (histograms) of selected metal lines in the $z_{\text {abs }}=2.08681$ DLA in Q1444+014. The $y$-axis is residual intensity. The velocity structure of this system is exceedingly complex; at least 13 separate absorption components (indicated by vertical tick marks in the top panel) contribute to the absorption lines. While the profile of Si II $\lambda 1304.4$ can be reproduced adequately by VPFIT (thin continuous line), we cannot derive reliable column densities for O I nor N I. In the former, the strongest components are heavily saturated, while the triplet structure of the latter leads to intractable blending. The VPFIT profiles for O I and N I are partial fits to the weaker components only, shown for illustration.

Table 4 where references to the original papers can also be found. All of the measurements in the table are from spectra of high signal-to-noise ratio and high (echelle) resolution obtained with $8-10 \mathrm{~m}$ class telescopes. For simplicity we limit ourselves to absorption systems with $N(\mathrm{H} \mathrm{I}) \geq 2 \times 10^{20} \mathrm{~cm}^{-2}$; inclusion of similar data available for a few sub-DLAs would not change any of our conclusions.

The full sample consists of 10 DLAs in which the abundance of $\mathrm{N}$ has been measured (seven detections and three upper limits). In one half of the cases the abundance of $\mathrm{O}$ is available directly, either because the O I $\lambda 1302.2$ line is not strongly saturated, or from weaker, unsaturated, transitions to higher energy levels. In the other five cases we take $N(\mathrm{~S}$ II) as a proxy for $N(\mathrm{O} \mathrm{I})$, after correcting for the solar abundance ratio $(\mathrm{O} / \mathrm{S})_{\odot}=+1.54$ (Grevesse \& Sauval 1998; Holweger 2001). The justification for this approach is as follows.

First, in Galactic stars the $(\mathrm{O} / \mathrm{S})$ ratio appears to be approximately solar over a wide range of metallicities down to $[\mathrm{O} / \mathrm{H}] \lesssim-2$ (Israelian et al. 2001; Israelian \& Rebolo 2001; Takada-Hidai et al. 2002; Nissen et al. in preparation). Second, 

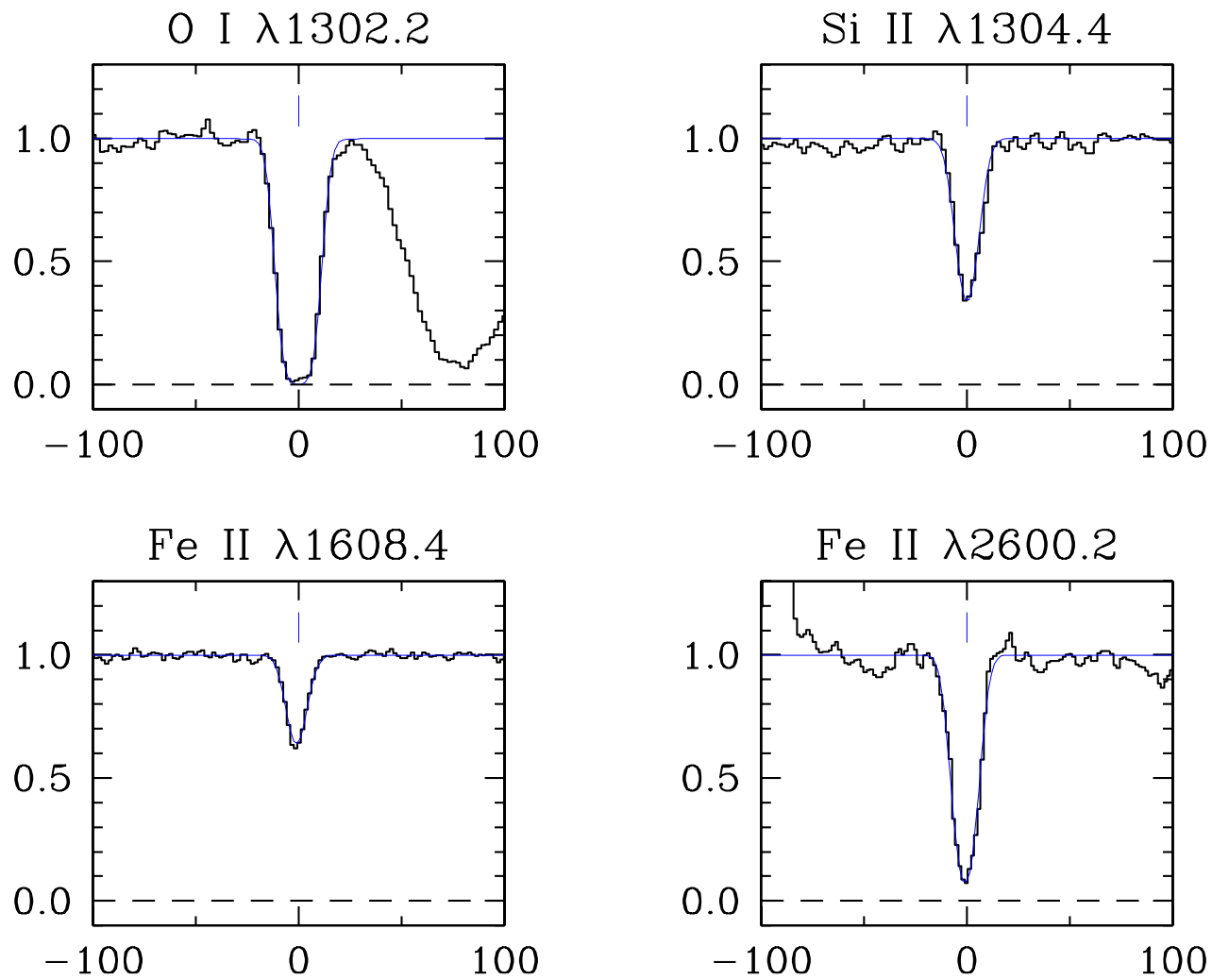

N I $\lambda \lambda 1199.5,1200.2,1200.7$

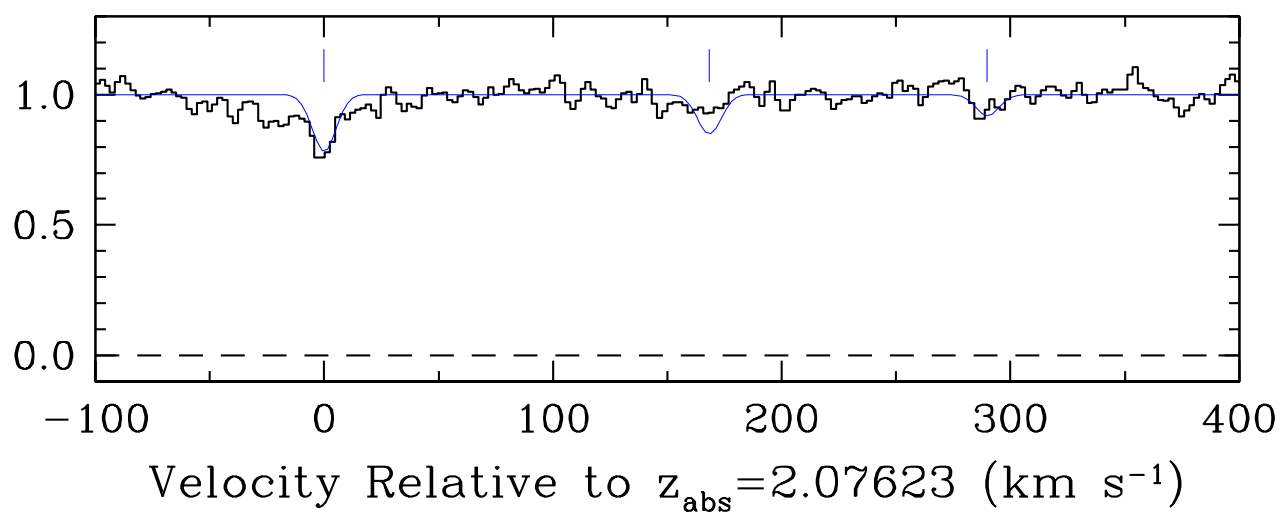

Fig. 5. Normalised absorption profiles (histograms) of selected metal lines in the $z_{\text {abs }}=2.07623$ DLA in Q2206-199. The $y$-axis is residual intensity. The thin continuous lines show theoretical profiles produced by VPFIT with the parameters listed in Table 2 . The absorption seen in this DLA is due to a single velocity component with $b=6.1 \mathrm{~km} \mathrm{~s}^{-1}$.

in the diffuse Galactic interstellar medium both elements show little affinity for dust (Savage \& Sembach 1996); dust corrections for $\mathrm{O}$ and $\mathrm{S}$ are almost certainly unimportant for our purposes at the reduced levels of dust depletions typical of metal-poor DLAs (Pettini et al. 1997; Vladilo 1998). Finally, differential ionisation corrections between O I and S II are likely to be small. Theoretically, the photoionisation calculations by Vladilo et al. (2001) show that, for all the models they considered, if $\log (\mathrm{O} / \mathrm{S})=\log [N(\mathrm{O} \mathrm{I}) / N(\mathrm{~S} \mathrm{II})]+C$, then $|C| \lesssim 0.2(\lessgtr 0.1)$ when $\log N(\mathrm{H} \mathrm{I}) \geq 20.2(\geq 20.6)$. This condition is met by 5 (4) out of the 5 DLAs in Table 4 for which we use $\mathrm{S}$ as a proxy for $\mathrm{O}$. For all the DLAs considered here $|C|[\mathrm{N} / \mathrm{O}] \leq 0.002$ so that $\log (\mathrm{N} / \mathrm{O})=\log [N(\mathrm{~N} \mathrm{I}) / N(\mathrm{O} \mathrm{I})]$.
Some authors (e.g. Lu et al. 1998) have also used $\mathrm{Si}$ to deduce the abundance of $\mathrm{O}$, by assuming that in DLAs $N(\mathrm{O} \mathrm{I}) / N(\mathrm{Si} \mathrm{II})=(\mathrm{O} / \mathrm{Si})_{\odot}$. This assumption, however, is less secure than the equivalent one for $\mathrm{S}$ and has been criticised, for example, by Matteucci et al. (1997). First, Si can be depleted onto dust. Second, it is unclear whether the abundances of $\mathrm{O}$ and Si do track each other at low metallicities (Edvardsson et al. 1993; Ryan et al. 1996; Israelian et al. 2001); theoretically they are not expected to, if some of the $\mathrm{Si}$ is produced by type Ia supernovae (Matteucci et al. 1997). Neither of these complications applies to $\mathrm{S}$. In the past, the use of $\mathrm{Si}$ as an indicator of the $\mathrm{O}$ abundance had been motivated by the paucity of measurements of $[\mathrm{O} / \mathrm{H}]$ and $[\mathrm{S} / \mathrm{H}]$ in DLAs; with the larger body 
Table 4. N, O, S, and Fe abundance measurements in DLAs.

\begin{tabular}{|c|c|c|c|c|c|c|c|c|c|}
\hline QSO & $z_{\mathrm{abs}}$ & $\begin{array}{c}\log N(\mathrm{H} \mathrm{I}) \\
\left(\mathrm{cm}^{-2}\right)\end{array}$ & $\begin{array}{c}\log N(\mathrm{~N} \mathrm{I}) \\
\left(\mathrm{cm}^{-2}\right)\end{array}$ & $\begin{array}{c}\log N(\mathrm{O} \mathrm{I}) \\
\left(\mathrm{cm}^{-2}\right)\end{array}$ & $\begin{array}{c}\log N(\mathrm{~S} \mathrm{II}) \\
\left(\mathrm{cm}^{-2}\right)\end{array}$ & $(\mathrm{O} / \mathrm{H})+12^{a}$ & $(\mathrm{~N} / \mathrm{O})^{a}$ & $(\mathrm{Fe} / \mathrm{O})^{a}$ & Ref. $^{b}$ \\
\hline Q0000-2620 & 3.3901 & 21.41 & 14.73 & 16.45 & $\ldots$ & 7.04 & -1.72 & -1.58 & 1 \\
\hline Q0100+1300 & 2.3090 & 21.32 & 15.29 & $\ldots$ & 15.12 & 7.34 & -1.37 & -1.56 & 2,3 \\
\hline Q0201+1120 & 3.38639 & 21.26 & 15.33 & $\ldots$ & 15.21 & 7.49 & -1.42 & -1.40 & 4 \\
\hline J0307-4945 & 4.46658 & 20.67 & 13.57 & 15.91 & $\ldots$ & 7.24 & -2.34 & -1.70 & 5 \\
\hline Q0347-3819 & 3.02486 & 20.63 & 14.89 & 16.64 & $\ldots$ & 8.01 & -1.75 & -2.21 & 6 \\
\hline Q1409+095 & 2.45620 & 20.54 & $\leq 13.19$ & 15.15 & $\ldots$ & 6.61 & $\leq-1.96$ & -1.41 & 7 \\
\hline GB1759+7539 & 2.62528 & 20.76 & 14.99 & $\ldots$ & $15.21^{c}$ & 7.99 & $-1.76^{c}$ & -1.81 & 8,9 \\
\hline Q2206-199 & 2.07623 & 20.44 & $\leq 12.88$ & 15.24 & $\ldots$ & 6.80 & $\leq-2.36$ & -1.87 & 7 \\
\hline Q2343+1230 & 2.4313 & 20.34 & 14.67 & $\ldots$ & 14.71 & 7.91 & -1.58 & $\ldots$ & 2 \\
\hline Q2348-1444 & 2.27936 & 20.56 & $\leq 13.22$ & $\ldots$ & 13.73 & 6.71 & $\leq-2.05$ & -1.48 & 3 \\
\hline
\end{tabular}

${ }^{a}$ When the oxygen abundance is not available, $\mathrm{S}$ has been used as a proxy for $\mathrm{O}$ by assuming the solar ratio $(\mathrm{O} / \mathrm{S})_{\odot}=+1.54(\mathrm{Grevesse} \&$ Sauval 1998; Holweger 2001); see text (Sect. 4) for justification.

${ }^{b}$ References - 1: Molaro et al. (2000); 2: Lu et al. (1998); 3: Prochaska \& Wolfe (1999); 4: Ellison et al. (2001); 5: Dessauges-Zavadsky et al. (2001); 6: Levshakov et al. (2002); 7: This work; 8: Outram et al. (1999); 9: Prochaska et al. (2002).

${ }^{c}$ Prochaska et al. (2002) found that only about half of the metals in this DLA arise in a neutral component, while the reminder are in partially ionised gas. It is unclear what fraction of S in the ionised gas is S II; in the extreme case that half of the observed S II is in an ionised region where $\mathrm{O} I$ and $\mathrm{N} I$ are absent, the value of $(\mathrm{N} / \mathrm{O})$ for this DLA should be increased by +0.3 dex.

of data now available this approach is no longer necessary. For this reason, we restrict ourselves to $\mathrm{O}$ and $\mathrm{S}$ in the following analysis.

\subsection{Nitrogen and oxygen in DLAs}

We are now ready to compare the abundances of $\mathrm{N}$ and $\mathrm{O}$ in high redshift DLAs with those measured in $\mathrm{H}$ II regions in the local universe (Fig. 6). For the latter, we use the compilation assembled by Henry et al. (2000 - references to the original surveys are given in the figure caption), augmented by some additional work (Ferguson et al. 1998; van Zee 2000). The dashed lines in the figure are approximate boundaries of the region in the $(\mathrm{N} / \mathrm{O})$ vs. $(\mathrm{O} / \mathrm{H})$ plot where we may expect DLAs to fall. The line labelled "Secondary" is simply an extrapolation to low $(\mathrm{O} / \mathrm{H})$ values of the $(\mathrm{N} / \mathrm{O})$ vs. $(\mathrm{O} / \mathrm{H})$ trend at high metallicities, where secondary $\mathrm{N}$ presumably dominates. If the extrapolation to low metallicities is valid (we do not know that it is), this is the minimum amount of $\mathrm{N}$ (relative to $\mathrm{O}$ ) which one may expect to find, irrespectively of the previous star formation history of the system under consideration. The "Primary" level is just the plateau at $(\mathrm{N} / \mathrm{O})=-1.5$ indicated by existing measurements in low metallicity $\mathrm{H}$ II regions. We stress that these are just approximate empirical limits which are not derived from a full chemical evolution treatment of the available data (e.g. Larsen et al. 2001)

As can be seen from Fig. 6, the DLAs in our sample do indeed populate the region of the diagram delimited by the broken lines. Six of the DLAs considered exhibit levels of $\mathrm{N}$ enrichment which are similar to those of the most metal-poor H II regions. On the other hand, in four cases $\mathrm{N}$ appears to be below the "Primary" line, by up to nearly one order of magnitude.

\section{Discussion}

The results presented here, in Fig. 6 and Table 4, confirm the earlier conclusion by Lu et al. (1998) that the (N/O) ratio in DLAs exhibits a larger range of values than that of any other pair of heavy elements which are not depleted onto dust (Prochaska \& Wolfe 2002). We now consider possible causes of this scatter.

The simplest explanation may be just measurement error. Although the errors on $N(\mathrm{~N} I)$ and $N(\mathrm{O}$ I $)$ quoted by the sources referenced in Table 4 are normally less than 0.1 dex (and always less than 0.2 dex), it must be remembered that the $\mathrm{N}$ I lines (a) are generally weaker than most other metal transitions used for abundance work in DLAs, and (b) occur within the Lyman $\alpha$ forest where blending can complicate their analysis. It is thus conceivable that the true uncertainties, particularly in the $\mathrm{N}$ abundance, may have been underestimated. On the other hand, if measurement error were the sole cause of the dispersion of the DLA points in Fig. 6, it would be a remarkable coincidence that all 10 values in our sample should fall within the boundaries delimited by the Primary and Secondary lines in the figure. In principle one would expect measurement errors to scatter points above the Primary line and, at the larger values of $(\mathrm{O} / \mathrm{H})$, below the Secondary boundary. For many of the low points in Fig. 6, it would be very hard to understand how an absorption line $\sim 3-5$ times stronger than observed could have been missed. We conclude, therefore, that measurement errors are not the principle cause of the dispersion observed.

We do not subscribe to the view proposed by Izotov et al. (2001) that unrecognised ionisation corrections are responsible for the low $(\mathrm{N} / \mathrm{O})$ values. As explained above, in about half of the cases $(\mathrm{N} / \mathrm{O})_{\text {DLA }}$ is comparable to the value measured in nearby metal-poor H II regions; there is no evidence that 


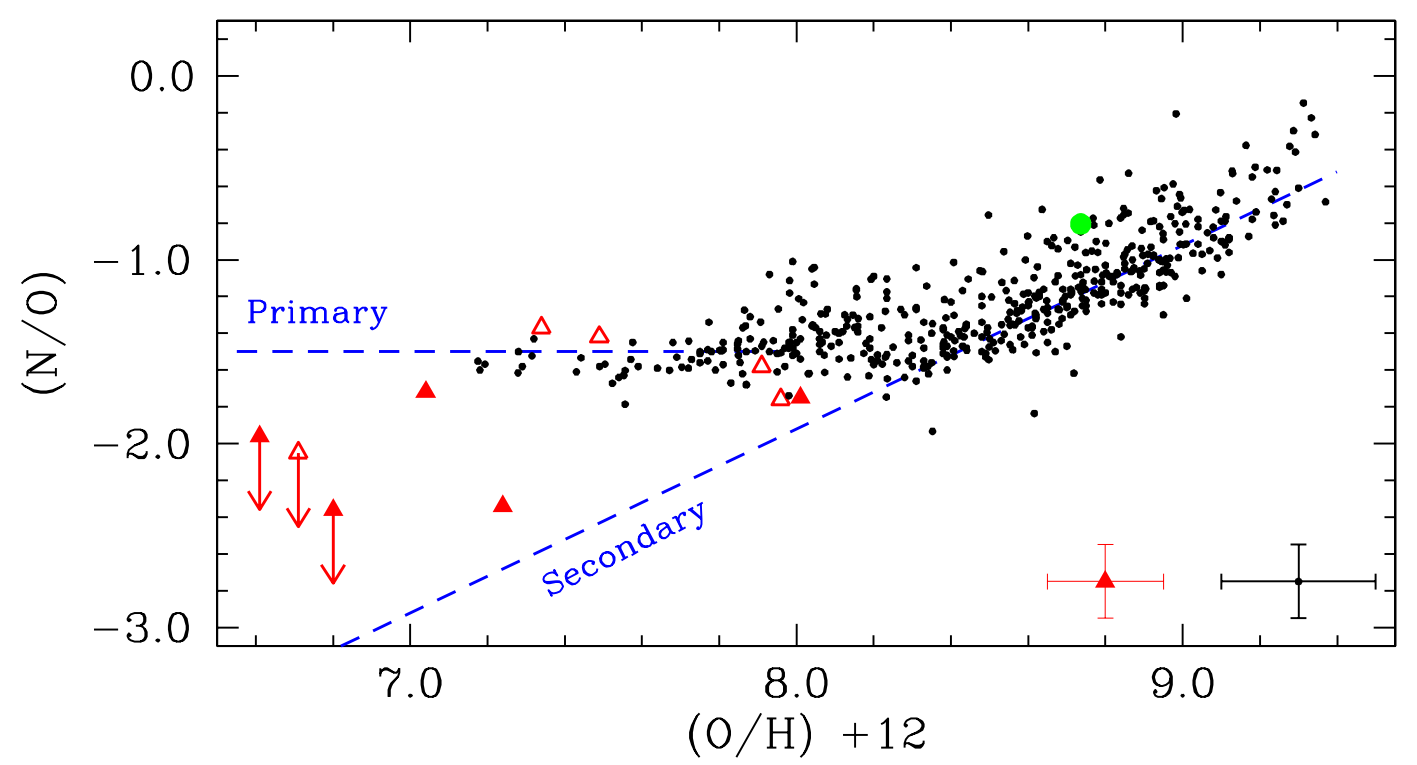

Fig. 6. Abundances of $\mathrm{N}$ and $\mathrm{O}$ in extragalactic $\mathrm{H}$ II regions (small dots) and damped Lyman $\alpha$ systems (large triangles). The $\mathrm{H}$ II region measurements are from the studies by Kobulnicky \& Skillman (1996); Thurston et al. (1996); van Zee et al. (1998); Ferguson et al. (1998); Izotov \& Thuan (1999); and van Zee (2000). The values for damped Lyman $\alpha$ systems are from the compilation in Table 4; filled triangles denote DLAs where the abundance of O could be measured directly, while open triangles are cases where $\mathrm{S}$ was used as a proxy for $\mathrm{O}$ (see text). The error bars in the bottom right-hand corner give an indication of the typical uncertainties; the large dot corresponds to the solar abundances of $\mathrm{N}$ and $\mathrm{O}$ from the recent reappraisal by Holweger (2001). The dashed lines are approximate representations of the primary and secondary levels of $\mathrm{N}$ production (see text).

these DLAs are different from the rest of the sample in their level of ionisation. Similarly, there is no separation in Fig. 6 between DLAs where $(\mathrm{O} / \mathrm{H})$ has been measured directly and those where it has been deduced by reference to $\mathrm{S}$.

Some may be concerned about possible systematic differences between abundances measured via absorption lines in cool interstellar $\mathrm{H}$ I clouds and those deduced from the analysis of nebular emission lines from H II gas. However, there are no precedents for such systematic differences in the solar vicinity (Meyer et al. 1997; Meyer et al. 1998) and, in any case, here we are dealing with a scatter rather than a systematic offset.

Qualitatively, our findings are consistent with, and indeed provide empirical evidence in favour of, an origin of primary nitrogen in intermediate mass stars. The observed range in the $(\mathrm{N} / \mathrm{O})$ ratio at low $(\mathrm{O} / \mathrm{H})$ is, in this picture, a natural consequence of the delayed release of $\mathrm{N}$ into the ISM relative to the $\mathrm{O}$ produced by Type II supernovae. However, there are puzzling aspects in this scenario too, in that the proportion of DLAs with values of $(\mathrm{N} / \mathrm{O})$ below the primary level seems at first sight to be surprisingly high. Recall that Henry et al. (2000) estimated the time delay between $\mathrm{N}$ and $\mathrm{O}$ release into the ISM to be $\sim 250 \mathrm{Myr}$, if the main source are stars in the mass range $7-4 M_{\odot}$. The median redshift of the DLA sample in Table 4 is $\langle z\rangle=2.5$; in a $\Omega_{\mathrm{M}}=0.3, \Omega_{\Lambda}=0.7, h=0.65$ cosmology this corresponds to a look-back time of $11.7 \mathrm{Gyr}^{3}$. There is now evidence that star formation in the universe was already at high levels well before this epoch, certainly by $z \gtrsim 4.5$ (Steidel et al. 1999), and possibly up to $z \simeq 6$ (Shapley et al. 2001; Becker et al. 2001; Dawson et al. 2001). In this cosmology, $z=6$ corresponds to a look-back time of $\sim 13.5 \mathrm{Gyr}$. Thus, the time

\footnotetext{
3 The age of the universe in this cosmology is $14.5 \mathrm{Gyr}$.
}

interval between $z=6$ and our median $\left\langle z_{\mathrm{DLA}}\right\rangle=2.5$ is seven times higher than the estimated time delay for the release of primary nitrogen. If the galaxies giving rise to DLAs formed continuously between $z=6$ and 2.5 , naively one may expect to find only one DLA out of seven in the interim period when primary nitrogen has not yet caught up with the oxygen released by the same episode of star formation. Instead we find at least four such cases out of ten (see Fig. 6).

Perhaps we are just seeing the effects of small number statistics and a larger sample of $(\mathrm{N} / \mathrm{O})$ measurements will in future reveal that the present $40 \%$ is an overestimate of the number of DLAs with a $\mathrm{N}$ deficiency. However, if such a high fraction is confirmed by future observations, we can think of two explanations for this apparent puzzle. The four DLAs with values of $(\mathrm{N} / \mathrm{O})$ clearly below the primary level in Fig. 6 have abundances $(\mathrm{O} / \mathrm{H})$ less than $\sim 1 / 30$ of solar. Thus, they have presumably experienced little star formation up to the time when we observe them; in a closed box model, they would have turned less than $1 / 30$ of their gas into stars. It therefore seems at least plausible that these DLAs arise preferentially in young galaxies, which have only recently condensed out of the intergalactic medium. In other words, the very fact that the difference between primary and secondary nitrogen is most pronounced at low metallicities introduces a bias in our sample such that it may include a relatively high proportion of galaxies caught in the initial stages of their chemical evolution (e.g. Cen et al. 2002).

A second possibility is that the time delay for the release of primary nitrogen may be longer when metal abundances are lower. This dependence may arise if at lower metallicities stars of progressively lower masses can synthesize and 
release nitrogen, because their interiors are hotter (Lattanzio \& Forestini 1999) and/or through the increasing importance of rotation (Meynet \& Maeder 2002a,b; see also Marigo 2001). The slope of the initial mass function would then shift the peak of the $\mathrm{N}$ production to lower mass stars, with longer evolutionary times. While these effects remain to be fully quantified, there have been suggestions that at the metallicities of DLAs the full release of primary $\mathrm{N}$ may lag behind that of $\mathrm{O}$ by up to $800 \mathrm{Myr}$ (Lattanzio et al., in preparation). If confirmed, this proposal would go a long way towards explaining the high proportion of DLAs which are underabundant in $\mathrm{N}$ at $\langle z\rangle=2.5$.

\subsection{Clues from the abundance of iron?}

It should be possible to test the hypothesis that the dispersion of $(\mathrm{N} / \mathrm{O})$ values in DLAs is a consequence of the delayed release of primary nitrogen by considering the abundances of other elements synthesized by low mass stars. Observationally, iron is one of the more easily accessible through several rest frame ultraviolet transitions. Measurements of $(\mathrm{Fe} / \mathrm{H})$ are available for 9 out of the 10 DLAs in our sample ${ }^{4}$; the corresponding values of $(\mathrm{Fe} / \mathrm{O})$ are listed in Table 4.

It is generally thought that approximately $2 / 3$ of the iron is produced in Type Ia supernovae. While it is still unclear which types of binary system give rise to such events, there seems to be a consensus that the evolutionary timescales of Type Ia SN progenitors are of the same order as, or longer than, those of the 7-4 $M_{\odot}$ stars which are the source of primary nitrogen (e.g. Matteucci \& Recchi 2001). From this it follows that DLAs deficient in $\mathrm{N}$ (in the sense of having less $\mathrm{N}$ than the primary level) should also exhibit sub-solar $(\mathrm{Fe} / \mathrm{O})$ ratios. We examine this point in Fig. 7, by plotting the quantity [(N/O) + 1.5] (that is, the difference between the measured $(\mathrm{N} / \mathrm{O})$ and the primary plateau at $(\mathrm{N} / \mathrm{O})=-1.5)$ vs. $[(\mathrm{Fe} / \mathrm{O})+1.29]$ (the difference between observed and solar $(\mathrm{Fe} / \mathrm{O})$ adopting $(\mathrm{Fe} / \mathrm{O})_{\odot}=-1.29$ from Holweger 2001).

There are two complications which affect the interpretation of the data in Fig. 7. First, if massive stars which explode as Type II SN also produce some Fe, the effect of time delay will be less pronounced for Fe than for N. Indeed, Galactic stars with metallicities similar to those of the DLAs considered here, between $\sim 1 / 10$ and $\sim 1 / 100$ of solar, are deficient in $\mathrm{Fe}$ only by factors of $2-3$. In the units of Fig. 7, $[\mathrm{Fe} / \mathrm{O}] \equiv(\mathrm{Fe} / \mathrm{O})-(\mathrm{Fe} / \mathrm{O})_{\odot}=-0.3$ to -0.5 (e.g. Israelian et al. 2001). This is larger, but not by much, than the typical accuracy of $0.1-0.2$ dex with which this ratio is measured in DLAs. A more serious problem is that, unlike $\mathrm{O}, \mathrm{Fe}$ can be depleted onto dust and this effect will mimic the intrinsic underabundance which we are testing for. The combination of the subtlety of the effect with the uncertainty in correcting for dust depletions may well explain, at least in part, why the $\alpha$-element enhancement well established in Galactic metal-poor stars has proved so difficult to pin down in DLAs (Vladilo 1998; Pettini et al. 2000; Prochaska \& Wolfe 2002; Ledoux et al. 2002). Accounting for

\footnotetext{
${ }^{4}$ The same is not true for other Fe-peak elements, especially $\mathrm{Zn}$ whose abundance may be easier to interpret because, unlike $\mathrm{Fe}, \mathrm{Zn}$ is not depleted onto dust.
}

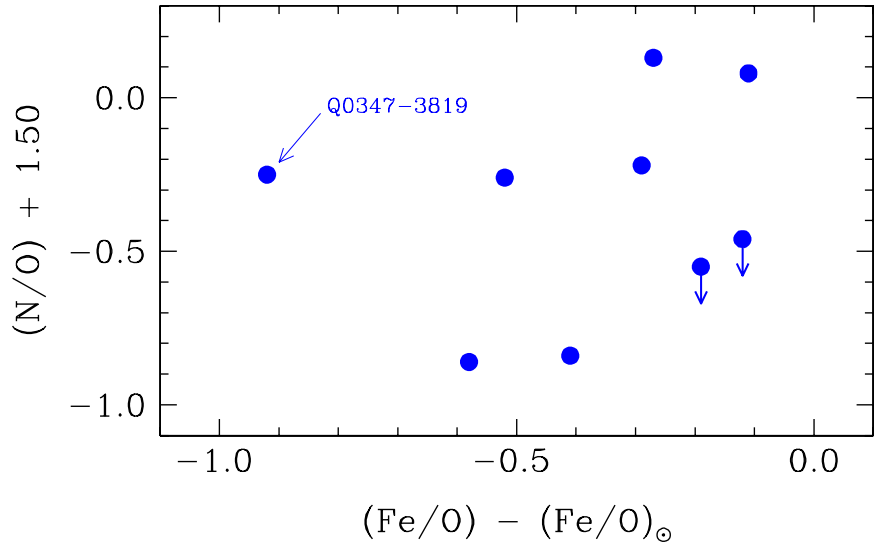

Fig. 7. In this figure we plot the deficiency of $\mathrm{N}$ relative to the Primary level at $(\mathrm{N} / \mathrm{O})=-1.5$ vs. $[\mathrm{Fe} / \mathrm{O}]$, the departure of the $(\mathrm{Fe} / \mathrm{O})$ ratio in DLAs from the solar $(\mathrm{Fe} / \mathrm{O})_{\odot}=-1.29$ (Holweger 2001). In the logarithmic units used here, the abundance ratio of two elements has a typical measurement error of between 0.1 and 0.2 . Measured values of $(\mathrm{N} / \mathrm{O})$ and $(\mathrm{Fe} / \mathrm{O})$ for the nine DLAs shown are listed in Cols. 8 and 9 of Table 4.

the fraction of $\mathrm{Fe}$ in solid form requires taking into consideration the full complement of abundance measures in a DLA (e.g. Vladilo 2002), and is beyond the scope of this work. The values of $[\mathrm{Fe} / \mathrm{O}]$ plotted in Fig. 7 should therefore be considered as indicative of the maximum intrinsic deficiency of Fe relative to $\mathrm{O}$ in the DLAs in our sample.

These complications make it difficult to draw firm conclusions from the results in Fig. 7. Some of the DLAs appear to meet our expectations, by exhibiting low values of $(\mathrm{N} / \mathrm{O})$ matched by corresponding Fe underabundances. Similarly, DLAs which lie closer to the Primary line at $(\mathrm{N} / \mathrm{O})=-1.5$ show a range of $[\mathrm{Fe} / \mathrm{O}]$ values, as expected if the time delay for the release of $\mathrm{Fe}$ is longer than that of $\mathrm{N}$. But there are also cases which evidently do not fit this pattern. The DLA in Q0347-3819 may be a special case, in that it is the only DLA known to date with such a strong $\alpha$-element enhancement (Levshakov et al. 2002). More problematic, however, are the two DLAs with low (N/O) (both are upper limits) and only a minor (if any) Fe deficiency. Possibly we have underestimated the $\mathrm{N}$ abundance in these cases because of blending with Lyman $\alpha$ forest lines 5 .

We conclude that the results of Fig. 7 do not provide clear support for the scenario where both $\mathrm{N}$ and Fe lag behind the release of $\mathrm{O}$ into the ISM. Possibly, as more data become available, a trend between $(\mathrm{N} / \mathrm{O})$ and $(\mathrm{Fe} / \mathrm{O})$ will emerge, if one exists. But Fig. 7 may also be telling us that there is a limit to

\footnotetext{
5 A similar apparent inconsistency was pointed out by Centurión et al. (1998) for the DLA in Q0100+1300. The HIRES observations of this QSO by Lu et al. (1998) and Prochaska \& Wolfe (1999), used here, give $(\mathrm{N} / \mathrm{O})+1.5=+0.13$ and $[\mathrm{Fe} / \mathrm{O}]=-0.27$, which are consistent with our working hypothesis. Centurión et al. (1998), on the other hand, derived a much lower column density of $\mathrm{N}-$ by a factor of four - from observations of the weaker N I $\lambda \lambda 1134$ triplet with the $4 \mathrm{~m}$ William Herschel telescope on la Palma. Observations of the $\mathrm{N}$ I $\lambda \lambda 1134$ triplet with an $8-10 \mathrm{~m}$ telescope are required to resolve this discrepancy.
} 
the degree to which our ideas on the chemical evolution of the Milky Way can be applied to the high redshift DLA galaxies.

\section{Summary and conclusions}

We have presented new measurements of the abundances of $\mathrm{N}$, $\mathrm{O}, \mathrm{Si}$, and $\mathrm{Fe}$ in two metal-poor damped Lyman $\alpha$ systems (plus one sub-DLA) obtained with UVES on the VLT. We have combined these data with others from the literature to form a sample of 10 DLAs for which reliable measurements of the abundance of $\mathrm{N}$ (or useful upper limits) are available. The sample consists exclusively of high resolution, high $\mathrm{S} / \mathrm{N}$ measurements obtained with $8-10 \mathrm{~m}$ class telescopes. Our aim was to test current ideas on the nucleosynthesis of $\mathrm{N}$, by extending measurements of the abundances of $\mathrm{N}$ and $\mathrm{O}$ from $\mathrm{H}$ II regions in the local universe to the younger and less enriched galaxies at high redshift $(\langle z\rangle=2.5)$ that give rise to the DLAs. Our principal conclusions are as follows.

1. The $(\mathrm{N} / \mathrm{O})$ ratio remains a difficult quantity to measure, because the absorption lines available generally have widely different optical depths, so that when the N I $\lambda 1200$ triplet is detected O I $\lambda 1302.2$ is saturated. Furthermore, the N I lines are often blended within the Lyman $\alpha$ forest. We have not identified an optimum strategy for dealing with these difficulties. DLAs at higher redshifts than those shown here make accessible weaker transitions of O I at far-UV wavelengths, but the chances of confusion of $\mathrm{N}$ I with Lyman $\alpha$ forest lines are then increased.

2. We have argued that $S$, when available, can be used as a proxy for $\mathrm{O}$, because they are both undepleted $\alpha$-elements and the differential ionisation corrections between $\mathrm{O} I$ and $\mathrm{S}$ II should not be important when the column density of neutral hydrogen is as high as in DLAs. However, it would obviously be reassuring to confirm empirically that $(\mathrm{O} / \mathrm{S}) \equiv N(\mathrm{O} \mathrm{I}) / N(\mathrm{~S}$ II $)$ in at least a few cases, with specially targeted observations.

3. We confirm earlier reports that the $(\mathrm{N} / \mathrm{O})$ ratio shows a larger dispersion of values than other ratios of heavy elements in DLAs. All 10 measurements in our sample fall within the region in the $(\mathrm{N} / \mathrm{O})$ vs. $(\mathrm{O} / \mathrm{H})$ plot bounded by the primary and secondary levels of $\mathrm{N}$ production.

4. We have considered several possible explanations for this scatter and conclude that the most plausible one is that we are seeing the effects of a time delay, by about $250 \mathrm{Myr}$, in the release of primary $\mathrm{N}$ from $7-4 M_{\odot}$ stars relative to the massive stars which release $\mathrm{O}$ when they explode as Type II supernovae. Thus, our results provide empirical evidence in support of currently favoured ideas for the nucleosynthesis of $\mathrm{N}$. The uniform value $(\mathrm{N} / \mathrm{O}) \simeq-1.5$ (on a log scale) seen in nearby metal-poor star-forming galaxies can be understood in this scenario if these galaxies are not young, but contain older stellar populations, as indicated by a number of imaging studies with HST.

5. A surprisingly high proportion (40\%) of DLAs in our sample have apparently not yet attained the full primary level of $\mathrm{N}$ enrichment at $(\mathrm{N} / \mathrm{O}) \simeq-1.5$. Possibly, the low metallicity regime - where the difference between secondary and primary nitrogen enrichment is most pronounced - preferentially selects young galaxies which have only recently condensed out of the intergalactic medium and begun forming stars. A more speculative alternative, which needs to be explored computationally, is that at low metallicities stars with masses lower than $4 M_{\odot}$ may make a significant contribution to the overall $\mathrm{N}$ yield. The release of primary $\mathrm{N}$ may, under these circumstances, continue for longer than $250 \mathrm{Myr}$, perhaps for a substantial fraction of the Hubble time at the median $\langle z\rangle=2.5$ of our sample.

6. In this scenario, the abundance of $\mathrm{Fe}$ - two thirds of which is presumed to originate from Type Ia supernovae - should also lag behind that of $\mathrm{O}$, by at least as long as that of $\mathrm{N}$. However, conflicting clues are provided by a plot (N/O) vs. $(\mathrm{Fe} / \mathrm{O})$ for the present data; while some DLAs do match the predictions of our working hypothesis, there also appear to be some deviant cases. The interpretation is complicated by the fact that some fraction of the $\mathrm{Fe}$ is also produced by massive stars and that depletion onto dust can be an issue. A larger sample of measurements is required to assess whether the relative abundances of $\mathrm{N}, \mathrm{O}$ and Fe in DLAs are consistent with current ideas of chemical evolution in galaxies.

7. Finally, we point out that the large dispersion in the abundance of $\mathrm{N}$ at these low metallicities essentially precludes the use of the $\mathrm{N} \mathrm{V} \lambda \lambda 1238,1242$ doublet in constraining models of the ionisation of the intergalactic medium from the ratios of different ions in the Lyman $\alpha$ forest (e.g. Boksenberg et al., in preparation).

Acknowledgements. It is a pleasure to acknowledge the competent assistance with the observations by the ESO support staff at Paranal. We are very grateful to Dick Henry for kindly providing us with electronic tables of most of the data (on local H II region abundances) plotted in Fig. 6, and to Jason X. Prochaska for making widely available his comprehensive compilation of DLA measurements and related atomic parameters. Special thanks are due to David Bowen for running his Monte Carlo simulations on our behalf. Mike Edmunds, Paolo Molaro, Bernard Pagel and Sam Rix made valuable comments on early versions of the manuscript. This work was supported in part by the European RTN programme "The Intergalactic Medium". Max Pettini thanks the Instituto de Astrofísica de Canarias and the Instituto de Astronomía, UNAM for their hospitality during visits when this work was completed.

\section{References}

Becker, R. H., Fan, X., White, R. L., et al. 2001, AJ, 122, 2850

Bowen, D. V., Blades, J. C., \& Pettini, M. 1995, ApJ, 448, 634

Bowen, D. V., Pettini, M., \& Blades, J. C. 2002, ApJ, in press

Cen, R., Ostriker, J. P., Prochaska, J. X., \& Wolfe, A. M. 2002, ApJ, submitted [astro-ph/0203524]

Centurión, M., Bonifacio, P., Molaro, P., \& Vladilo, G. 1998, ApJ, 509,620

Contini, T., Treyer, M. A., Sullivan, M., \& Ellis, R. S. 2002, MNRAS, 330,75

Crone, M. M., Schulte-Ladbeck, R. E., Greggio, L., \& Hopp, U. 2002, ApJ, 567, 258

Dawson, S., Stern, D., Bunker, A. J., Spinrad, H., \& Dey, A. 2001, AJ, 122,598 
Dekker, H., D’Odorico, S., Kaufer, A., Delabre, B., \& Kotzlowski, H. 2000, Proc. SPIE Conf., 4008, 534

Dessauges-Zavadsky, M., D’Odorico, S., McMahon, R. G., et al. 2001, A\&A, 370, 426

D'Odorico, S., Cristiani, S., Dekker, H., et al. 2000, Proc. SPIE Conf., 4005, 121

Edmunds, M. G., \& Pagel, B. E. J. 1978, MNRAS, 185, 77P

Edvardsson, B., Andersen, J., Gustafsson, B., et al. 1993, A\&A, 275, 101

Ellison, S. L., Pettini, M., Steidel, C. C., \& Shapley, A. E. 2001, ApJ, 549,770

Ellison, S. L., Ryan, S. G., \& Prochaska, J. X. 2001, MNRAS, 326, 628

Ferguson, A. M. N., Gallagher, J. S., \& Wyse, R. F. G. 1998, AJ, 116, 673

Grevesse, N., \& Sauval, A. J. 1998, Space Sci. Rev., 85, 161

Henry, R. B. C., Edmunds, M. G., \& Köppen, J. 2000, ApJ, 541, 660

Holweger, H. 2001, in Solar and Galactic Composition, ed. R.F. Wimmer-Schweingruber (Berlin: Springer), 23

Israelian, G., \& Rebolo, R. 2001, ApJ, 557, L43

Israelian, G., Rebolo, R., García López, R. J., et al. 2001, ApJ, 551, 833

Izotov, Y., et al. 2001, in Gaseous Matter in Galaxies and Intergalactic Space, ed. R. Ferlet, M. Lemoine, J. M. Desert, \& B. Raban (Frontier Group), 225

Izotov, Y., Schaerer, D., \& Charbonnel, C. 2001, ApJ, 549, 878

Izotov, Y. I., \& Thuan, T. X. 1999, ApJ, 511, 639

Kobulnicky, H. A., \& Skillman, E. D. 1996, ApJ, 471, 211

Larsen, T. I., Sommer-Larsen, J., \& Pagel, B. E. J. 2001, MNRAS, 323,555

Lattanzio, J. C., \& Forestini, M. F. 1999, in Asymptotic Giant Branch Stars, ed. T. Le Bertre, A. Lébre, \& C. Waelkens, IAU Symp., 191, 31

Ledoux, C., Bergeron, J., \& Petitjean, P. 2002, A\&A, 385, 802

Levshakov, S. A., Dessauges-Zavadsky, M., D’Odorico, S., \& Molaro, P. 2002, ApJ, 565, 696

Lu, L., Sargent, W. L. W., \& Barlow, T. A. 1998, AJ, 115, 55

Maeder, A. 1992, A\&A, 264, 105

Marigo, P. 2001, A\&A, 370, 194

Matteucci, F., Molaro, P., \& Vladilo, G. 1997, A\&A, 321, 45

Matteucci, F., \& Recchi, S. 2001, ApJ, 558, 351

Meyer, D. M., Cardelli, J. A., \& Sofia, U. J. 1997, ApJ, 490, L103

Meyer, D. M., Jura, M., \& Cardelli, J. A. 1998, ApJ, 493, 222

Meynet, G., \& Maeder, A. 2002a, A\&A, 381, L25
Meynet, G., \& Maeder, A. 2002b, A\&A, submitted

Molaro, P., Bonifacio, P., Centurión, M., et al. 2000, ApJ, 541, 54

Outram, P. J., Chaffee, F. H., \& Carswell, R. F. 1999, MNRAS, 310, 289

Pettini, M., \& Bowen, D. V. 2001, ApJ, 560, 41

Pettini, M., Ellison, S. L., Steidel, C. C., \& Bowen, D. V. 1999, ApJ, 510, 576

Pettini, M., Ellison, S. L., Steidel, C. C., Shapley, A. E., \& Bowen, D. V. 2000, ApJ, 532, 65

Pettini, M., \& Hunstead, R. W. 1990, Aust. J. Phys., 43, 227

Pettini, M., Hunstead, R. W., Smith, L. J., \& Mar, D. P. 1990, MNRAS, 246, 545

Pettini, M., King, D. L., Smith, L. J., \& Hunstead, R. W. 1997, ApJ, 478, 536

Pettini, M., Lipman, K., \& Hunstead, R. W. 1995, ApJ, 451, 100

Pilyugin, L. S. 1999, A\&A, 346, 428

Prochaska, J. X., Howk, J. C., O'Meara, J. M., et al. 2002, ApJ, 571, 693

Prochaska, J. X., \& Wolfe, A. M. 1997, ApJ, 474, 140

Prochaska, J. X., \& Wolfe, A. M. 1999, ApJS, 121, 369

Prochaska, J. X., \& Wolfe, A. M. 2002, ApJ, 566, 68

Prochaska, J. X., Wolfe, A. M., Tytler, D., et al. 2001, ApJS, 137, 21

Ryan, S .G., Norris, J. E., \& Beers, T. C. 1996, ApJ, 471, 254

Savage, B. D., \& Sembach, K. R. 1996, ARA\&A, 34, 279

Schulte-Ladbeck, R. E., Hopp, U., Greggio, L., et al. 2001, in Dwarf Galaxies and Their Environment, ed. K. S. de Boer, R. J. Dettmar, \& U. Klein (Aachen: Shaker), 83

Shapley, A. E., Steidel, C. C., Adelberger, K. L., et al. 2001, ApJ, 562, 95

Steidel, C. C., Adelberger, K. L., Giavalisco, M., Dickinson, M., \& Pettini, M. 1999, ApJ, 519, 1

Takada-Hidai, M., Takeda, Y., Sato, S., et al. 2002, ApJ, 573, 614

Thurston, T. R., Edmunds, M. G., \& Henry, R. B. C. 1996, MNRAS, 283, 990

van den Hoek, L. B., \& Groenewegen, M. A. T. 1997, A\&AS, 123, 305

van Zee, L. 2000, ApJ, 543, L31

van Zee, L., Salzer, J. J., Haynes, M. P., O’Donoghue, A. A., \& Balonek, T. J. 1998, AJ, 116, 2805

Vladilo, G. 1998, ApJ, 493, 583

Vladilo, G. 2002, ApJ, 569, 295

Vladilo, G., Centurión, M., Bonifacio, P., \& Howk, J. C. 2001, ApJ, 557,1007 